\title{
1 Running and knee osteoarthritis: a systematic review and meta-analysis
}

\section{Abstract}

\section{Background}

4 Osteoarthritis $(\mathrm{OA})$ is a chronic condition characterised by pain, impaired function and reduced quality of life. A number of risk factors for knee OA have been identified such as obesity, occupation and injury. The association between physical activity or particular sports such as running and knee $\mathrm{OA}$ is less clear. Previous reviews, and the evidence which informs them, present contradictory or inconclusive findings.

Purpose

This systematic review aimed to determine the association between running and the development of knee OA.

\section{Study Design}

Systematic review and meta-analysis.

\section{Method}

Four electronic databases were searched, along with citations in eligible articles and reviews, and the contents of recent journal issues. Two reviewers independently screened the titles and abstracts

17 using pre-specified eligibility criteria. Full-text articles were also independently assessed for eligibility. Eligible studies were those in which running or running-related sports (e.g. triathlon or orienteering) were assessed as a risk factor for the onset or progression of knee OA in adults. Relevant outcomes included 1) diagnosis of knee $O A, 2)$ radiographic markers of knee $O A, 3)$ knee

21 joint surgery for $\mathrm{OA}$, 4) knee pain or 5) knee-associated disability. Risk of bias was judged using the

22 Newcastle-Ottowa scale. A random-effects meta-analysis was performed with case-control studies 23 investigating arthroplasty. 
After de-duplication, the search returned 1322 records. 153 full-text articles were assessed. 25 were eligible, describing 15 studies: 11 cohort (6 retrospective) and 4 case-control studies. Findings of studies with a diagnostic OA outcome were mixed. There were some radiographic differences observed in runners, but only at baseline within some subgroups. Meta-analysis suggested a protective effect of running against surgery due to OA: pooled OR $0.46(95 \% \mathrm{Cl} 0.30,0.71)$. The $\mathrm{I}^{2}$ was $0 \%(95 \% \mathrm{Cl} 0 \%, 73 \%)$. Evidence relating to symptomatic outcomes was sparse and inconclusive.

\section{Conclusion}

On this evidence, it is not possible to conclude the role of running in knee OA. Moderate to low quality evidence suggests: no association with OA diagnosis, a positive association with OA diagnosis, and a negative association with knee OA surgery. Conflicting results may reflect methodological heterogeneity. More well-designed, prospective evidence is needed to clarify the contradictions.

\section{Keywords}

Osteoarthritis, Running, Physical activity, Knee joint, Systematic review

What is known about the subject

The conclusions of previous reviews exploring the role of sport and physical activity in the development of knee osteoarthritis are inconsistent. The knee joint structures may respond differently to different types of physical activity, but it is unclear what the effect of running may be.

What this study adds to existing knowledge

This systematic review offers a comprehensive and up-to-date synthesis of the evidence surrounding running and knee $\mathrm{OA}$, incorporating a number of $\mathrm{OA}$ related markers and symptoms. Scant, low quality prospective evidence suggests either no association or an increased risk of diagnosis. On the 

running against knee replacement surgery.

48 
Osteoarthritis $(O A)$ is a chronic condition that is characterised by pain, impaired function and reduced quality of life. In the US, estimates suggest almost 27 million adults have clinically diagnosed $\mathrm{OA}^{30}$. An estimated 3.5 million people over the age of 50 in the UK currently have disabling $\mathrm{OA}^{48}$. The knee is one of the most commonly affected joints ${ }^{31}$, with over 9 million people estimated to have knee OA in the US ${ }^{30}$. Despite significant progress over recent decades, much remains unknown regarding the aetiology of knee OA. A number of risk factors have been identified, such as obesity, occupational activity level and joint injury ${ }^{3}$. Other factors which have been demonstrated to influence OA susceptibility include age, gender, genetics and ethnicity ${ }^{17}$. The association between physical activity or exercise and knee $O A$ is less clear.

It has been postulated that OA develops following either excessive physiological loading on normal joint structures, or normal loading on compromised structures (following injury, for example) ${ }^{11}$. OA is a mechanically driven condition ${ }^{41}$. How the individual knee structures respond to dynamic, cyclical loading patterns during running (particularly over prolonged periods) is unclear. If the mechanical loading stimulus of running helps elicit beneficial adaptation to the joints and surrounding structures, it may have a protective effect. Conversely, if a joint's tolerance to loading is exceeded as a result of running, it could be a risk factor. The relationship is further complicated as running itself is directly (and indirectly) associated with other risk factors such as joint injury and $\mathrm{BMI}^{3,49}$. There is variation in the risk of knee joint injury across different sports and physical activities ${ }^{22,32}$. Therefore, studying running independently from other sports may help to understand the relationship between physical activity and OA risk.

A number of reviews have investigated the role of physical activity, or particular sports, in the development of $\mathrm{OA}$ and have been inconclusive or contradictory ${ }^{3,8,50,51}$. One explanation for discrepant conclusions may lie in the different methods used by studies to measure and classify physical activity. ${ }^{3}$ The review by Urquhart et al ${ }^{50}$, for example, excluded studies investigating 
physical activities of daily living. In addition, the type of sporting activity may be relevant, if different activities affect the knee joint structures in non-consistent ways ${ }^{6}$. Some previous reviews reported on the role of running in knee $\mathrm{OA}^{8,12,43}$. However, one of these is now over 10 years old ${ }^{43}$ whilst the two more recent reviews were restricted in scope: one examining elite-level running only ${ }^{12}$ and one was limited by language (English only) and date (post 1990) ${ }^{8}$. The objective of this review is to determine from the published literature what the role is of running in the development of knee OA.

\section{Methods}

Recommendations by the Cochrane Collaboration ${ }^{14}$ were adopted for this review. The protocol was registered on PROSPERO database (reg. no. CRD42015024001) ${ }^{4}$.

\section{Search strategy}

Four electronic databases (MEDLINE via OvidSP, Embase via OvidSP, SPORTDiscus via EBSCOhost, and PEDro (Physiotherapy Evidence Database)) were searched (for search terms see online appendix 1). Searches were not limited by language or date. Database searches were supplemented by handsearching the citations in identified reviews and eligible articles, as well as of the contents of recent/in-press editions of four pre-specified relevant journals (AJSM, JAMA, Osteoarthritis and Cartilage, Journal of Bone and Joint Surgery). Searches took place from June to November 2015, and results were imported and de-duplicated using EndNote X6.

\section{Study selection}

Two reviewers independently assessed each reference against pre-specified inclusion and exclusion criteria (see protocol on PROSPERO) using a two-stage process: firstly, titles and abstracts, and, secondly, full-text articles. Discrepancies were settled by discussion between reviewers or consultation with a third author. Eligible studies were cohort, case-control studies or randomised 
trials which included adult samples, measured exposure to any form of running or jogging (including running-related sports such as triathlon and orienteering), included a comparison group, and assessed the following outcomes:

1. any definition of diagnosed knee OA and/or

2. radiographic/imaging markers of knee $\mathrm{OA}$ and/or

3. knee arthroplasty for OA and/or

4. knee pain and/or

5. disability specifically associated with the knee.

Excluded studies were those that reported outcomes not specific to the knee joints, and those in which the time between exposure to running and the outcome was inadequate (a minimum of one year). Retrospective cohorts, defined as cohorts in which prior running exposure was established at recruitment, were eligible. Studies were also excluded where running exposure was combined with other sports or activities, therefore running exposure could not be identified independently. This review did not consider grey literature. More detailed eligibility criteria are available in the review protocol. Studies were not excluded on the basis of language or date. Translators were sought for non-English references.

Data extraction and synthesis

Data were extracted for each eligible article by a single reviewer, using a pre-piloted extraction form. Data extraction was checked by a second reviewer. Where multiple publications were found for a study, the most recent results for each outcome were extracted. Where a study included more than one comparator, comparisons with community controls were prioritised (over, for example, comparisons against athletes from other sports).

All eligible studies are included in a narrative synthesis, organised by outcome and study design. Meta-analysis was considered for each eligible outcome; however, due to high levels of between- 
study methodological heterogeneity and small numbers of studies for each outcome, meta-analysis

122

123

124

was appropriate for only one outcome: knee arthroplasty (case-control studies). Due to the observational nature of case-control studies, a random-effects model was conducted in RevMan, using the Mantel-Haenszel method of weighting ${ }^{34}$. All rates entered were crude (unadjusted). Missing data were not accounted for. Measurement effects are expressed as odds ratios (OR) with $95 \%$ confidence intervals $(\mathrm{Cl})$. Due to the small number of studies $(n=3)$, subgroup or sensitivity analyses (as pre-specified in the protocol) were not undertaken. The $I^{2}$ statistic was used as a measure of heterogeneity, with 95\% confidence intervals using the non-central Chi ${ }^{2}$ approach ${ }^{16}$.

Meta-analyses were performed using Review Manager (RevMan) version $5.3^{46}$.

\section{Risk of bias}

The Newcastle Ottawa scale ${ }^{53}$ was used to assess each eligible study for risk of bias. Two reviewers independently assessed each study. Disagreements in ratings were resolved by consensus or on consultation with a third reviewer. Studies were not excluded on the basis of risk of bias.

The possibility of publication bias cannot be excluded. Funnel plots were not attempted because there were too few studies included the meta-analysis ${ }^{45}$.

\section{RESULTS}

The search results are shown in Figure 1, according to PRISMA guidelines ${ }^{35}$. Following screening, 25 $\operatorname{articles}^{1}$ were identified as eligible, describing 15 studies. Study names were assigned comprising first author and year of first publication (see Table 1). Year of (first) publication ranged from 1977 to 2010. Two studies were not published in English: one was Danish ${ }^{7}$, one was German ${ }^{15}$.

\footnotetext{
${ }^{1}$ References \# 5, 7, 9-10, 15, 18-21, 23-29, 32, 35-40, 42, 45
} 
Figure 1: Flowchart of search results, adapted from PRISMA ${ }^{35}$

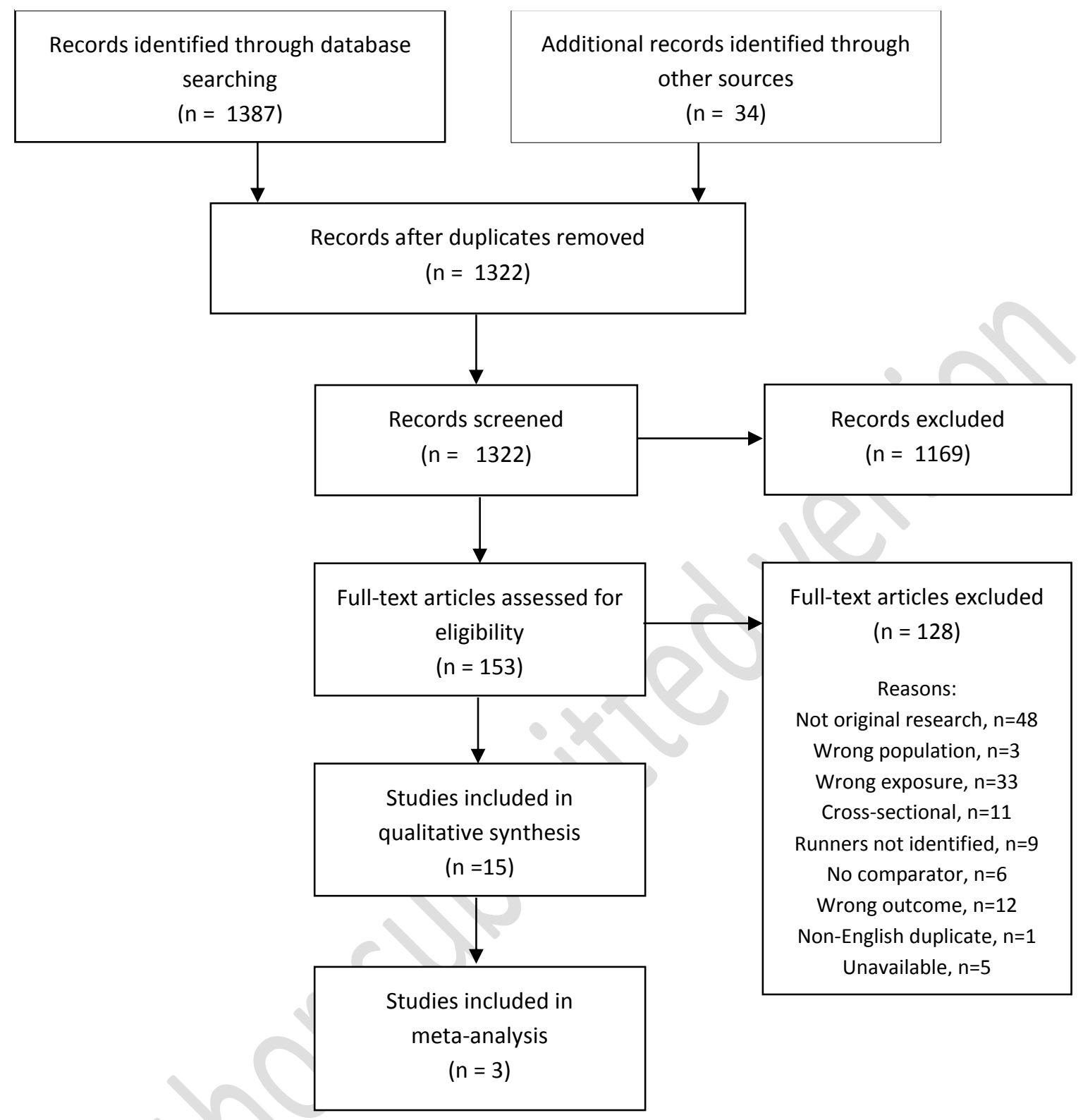

145 Study characteristics are summarised in Table 1. The majority $(n=11)$ were cohort studies, 6 of which 146 were retrospective ${ }^{7,15,21,36,37,44}$. The remaining 4 studies used a case-control design ${ }^{20,33,42,47}$. All of

147 the eligible studies were based in either European or USA populations. 
Three studies, all investigating radiographic outcomes, identified fewer than 5 years running exposure, and could be described as short-term ${ }^{15,36,37}$. Three studies were long-term (exposure and outcome separated by at least $25 y r)^{19,21,44}$, and four were medium-term (between 5 and 25 years' exposure $)^{5,10,25,39}$. One study ${ }^{7}$ did not report the study duration.

In the majority of the cohort studies ( $n=7)$, exposure to running was defined as membership of a club or association or as having taken part in competition. One cohort was recruited from a broader community, rather than via clubs or competition records ${ }^{10}$. Several studies ${ }^{15,37,38}$ did not describe recruitment nor how the exposure was determined.

Sample sizes of cohorts ranged from 15 to 1279 (see Table 1) with seven of the 11 cohorts including small $(n \leq 100)$ samples. Five of the cohorts included both males and females, one included only females ${ }^{44}$, and five studies included only males. Mean age at outcome assessment ranged from 27.4 to around 69 years. All but 3 of the studies investigated running or jogging as the exposure. The other 3 studies investigated orienteering or triathlon. No studies specifically reported on exposure to short-distance running.

Five of the cohorts recruited only elite athletes (or ex-professional athletes). Non-runners were recruited from a variety of sources: public military archives; the community; hospital radiology defined, identified or recruited ${ }^{37,38}$.

\section{Case control studies}

Three of the case control studies based their case definition on hospital registries of knee arthroplasty procedures: in Sweden ${ }^{42}$, Finland ${ }^{33}$ and the USA ${ }^{20}$. The other case control study ${ }^{47}$ was based in Sweden and used listed diagnosis of knee OA from hospital registers to define cases. To 
172 assess exposure to running (and other sports and activities), participants were mailed

173 questionnaires ${ }^{20,42,47}$ or were interviewed ${ }^{33}$.

174 The studies based in Finland and Sweden ${ }^{33,42,47}$ were able to randomly select controls from national

175 registers of the base population. The US-based study ${ }^{20}$ recruited controls from the Stanford Lipid

176 Research Study. Three of the case control studies matched on age and sex. Thelin 2006 additionally

177 matched on residency area. Sandmark 1999 did not report matching.

178 Two of the studies ${ }^{20,33}$ investigated running, whilst one study ${ }^{47}$ focussed on orienteering, and one

179 study ${ }^{42}$ measured both jogging and orienteering. All case-control studies included both males and

180 females.

181 
Table 1: Characteristics of included studies (chronological order of $1^{\text {st }}$ publication)

\begin{tabular}{|c|c|c|c|c|c|c|c|c|}
\hline Study ID & Study type & Sport(s) & $\begin{array}{l}\text { Sport } \\
\text { level }\end{array}$ & $\begin{array}{l}\text { Follow- } \\
\text { up (yr) }\end{array}$ & $\begin{array}{l}\text { Number of } \\
\text { participants }\end{array}$ & Exposed group/Cases, $\mathrm{n}$ & Non-exposed group/controls, $\mathrm{n}$ & $\begin{array}{l}\text { Exposure definition \& } \\
\text { measurement }\end{array}$ \\
\hline $\begin{array}{l}\text { de Carvalho } \\
1977^{7}\end{array}$ & $\begin{array}{l}\text { Retrospective } \\
\text { cohort }\end{array}$ & Running & NR & NR & 64 & $\begin{array}{l}\text { Runners recruited from club: } 100 \% \mathrm{M} \text {, } \\
\text { mean age } 47.7 \mathrm{yr}, \mathrm{n}=32\end{array}$ & $\begin{array}{l}\text { Radiology patients (excl hip/knee disorder or } \\
\text { arthritis): } 100 \% \text { M, age- and weight-matched, } \\
n=32\end{array}$ & $\begin{array}{l}\text { Membership of club } \\
\text { Mean yr running 23.9; } \\
\text { mean distance/wk } \\
33.65 \mathrm{~km}\end{array}$ \\
\hline $\begin{array}{l}\text { Lane } \\
1986^{5,26-29}\end{array}$ & $\begin{array}{l}\text { Prospective } \\
\text { cohort }\end{array}$ & Running & NR & 18 & 98 & $\begin{array}{l}\text { Runners recruited from club: } 56 \% M \text {, } \\
\text { mean age at recruitment } 57.5, n=45\end{array}$ & $\begin{array}{l}\text { Drawn from Stanford Lipid Research Study: } \\
56 \% \text { M, mean age } 57.7 \text {, age-, sex-, } \\
\text { occupation- and education-matched, } n=53\end{array}$ & $\begin{array}{l}\text { Membership of club OR } \\
\text { questionnaire-reported } \\
\text { current running }\end{array}$ \\
\hline $\begin{array}{l}\text { Panush } \\
1986^{38,39}\end{array}$ & $\begin{array}{l}\text { Ambispective* } \\
\text { cohort }\end{array}$ & Running & NR & $\begin{array}{l}5 \text { retro, } 8 \\
\text { prosp }\end{array}$ & 35 & $\begin{array}{l}\text { 'Runners': recruitment unclear, } 100 \% \\
\text { M, mean age at recruitment } 56, n=17\end{array}$ & $\begin{array}{l}\text { 'Controls': recruitment unclear, } 100 \% \mathrm{M} \text {, } \\
\text { mean age at recruitment } 61, n=18\end{array}$ & $\begin{array}{l}\text { 'Runners' ran } \geq 32 \mathrm{~km} / \mathrm{wk} \\
\text { for } \geq 5 \mathrm{yr}\end{array}$ \\
\hline $\begin{array}{l}\text { Kohatsu } \\
1990^{20}\end{array}$ & Case-control & Running & NR & $\mathrm{n} / \mathrm{a}$ & 92 & $\begin{array}{l}\text { Patients with TKR for severe knee OA } \\
\text { ( } \geq \text { Grade } 3+\text { history) from hospital } \\
\text { registers 1977-88: } 39 \% M \text {, mean age } \\
71.3, n=46\end{array}$ & $\begin{array}{l}\text { Drawn from Stanford Lipid Research Study: } \\
39 \% \text { M, mean age } 70.8 \text {, age- and sex- } \\
\text { matched, } n=46\end{array}$ & $\begin{array}{l}\text { Leisure-time running } \\
\text { assessed by mailed } \\
\text { questionnaire }\end{array}$ \\
\hline $\begin{array}{l}\text { Konradsen } \\
1990^{21}\end{array}$ & $\begin{array}{l}\text { Retrospective } \\
\text { cohort }\end{array}$ & Orienteering & $\begin{array}{l}\text { Competit } \\
\text { ive/elite }\end{array}$ & $\sim 35-40$ & 60 & $\begin{array}{l}\text { Qualifiers for county teams } 1950-55 \text { : } \\
100 \% M \text {, median age } 58, n=30\end{array}$ & $\begin{array}{l}\text { Radiology patients (abdominal): 'sedentary', } \\
100 \% \text { M, age-, height-, weight- and } \\
\text { occupational activity-matched, } n=30\end{array}$ & $\begin{array}{l}\text { Qualification for county } \\
\text { teams } 1950-55\end{array}$ \\
\hline $\begin{array}{l}\text { Kujala } \\
1994^{18,19,23,} \\
24,40\end{array}$ & $\begin{array}{l}\text { Ambispective* } \\
\text { cohort }\end{array}$ & $\begin{array}{l}\text { Long- } \\
\text { distance } \\
\text { running }\end{array}$ & $\begin{array}{l}\text { Competit } \\
\text { ive/elite }\end{array}$ & $\begin{array}{l}\sim 28-72 \\
\text { retro, } 3 \\
\text { prosp }\end{array}$ & 117 or & $\begin{array}{l}\text { Competitors in international events } \\
1920-65: 100 \% M, \text { mean age at } \\
\text { recruitment } 59.7, n=28 \text { or } 199^{* *}\end{array}$ & $\begin{array}{l}1 \text { Drawn from public archives: } 100 \% \mathrm{M} \text {, age- } \\
\text { and residence area-matched, } \mathrm{n}=1712^{19,23} \\
2 \text { Competitors in international events } 1920-65 \\
\text { (soccer, weight-lifting, shooting): } 100 \% \mathrm{M} \text {, } \\
\text { mean ages } 56.5,59.3,61, \mathrm{n}=89^{18,23,24,40}\end{array}$ & $\begin{array}{l}\text { Representation in } \\
\text { international competition } \\
\text { 1920-65 }\end{array}$ \\
\hline $\begin{array}{l}\text { Spector } \\
1996^{44}\end{array}$ & $\begin{array}{l}\text { Retrospective } \\
\text { cohort }\end{array}$ & $\begin{array}{l}\text { Middle- and } \\
\text { long-distance } \\
\text { running }\end{array}$ & $\begin{array}{l}\text { Competit } \\
\text { ive/elite }\end{array}$ & $\sim 20-46$ & & $\begin{array}{l}\text { Competitors in national/international } \\
\text { events 1950-79: } 0 \% \mathrm{M} \text {, mean age } 52.3\end{array}$ & $\begin{array}{l}1 \text { Drawn from Chingford cohort or twin study: } \\
0 \% \mathrm{M} \text {, mean age } 54.2, \mathrm{n}=977 \\
2 \text { Competitors in national/international tennis } \\
\text { events } 1950-79: 0 \% \mathrm{M} \text {, mean age } 52.3 \text {, } \\
\mathrm{n}=215\end{array}$ & $\begin{array}{l}\text { Representation in } \\
\text { international competition } \\
1950-79\end{array}$ \\
\hline $\begin{array}{l}\text { Kujala } \\
1999^{25}\end{array}$ & $\begin{array}{l}\text { Prospective } \\
\text { cohort }\end{array}$ & Orienteering & $\begin{array}{l}\text { Competit } \\
\text { ive/elite }\end{array}$ & & 529 & $\begin{array}{l}\text { Orienteers recruited from } 1984 \\
\text { ranking records: } 100 \% M \text {, mean age at } \\
\text { recruitment } 48.6, n=300\end{array}$ & $\begin{array}{l}\text { Drawn from public archives (excl obese, } \\
\text { smokers, CHD, OA): } 100 \% \text { M, mean age } 60.3, \\
\text { age- and residence area-matched, } n=229\end{array}$ & $\begin{array}{l}\text { Inclusion in national } \\
\text { ranking in } 1984\end{array}$ \\
\hline $\begin{array}{l}\text { Sandmark } \\
1999^{42}\end{array}$ & Case-control & $\begin{array}{l}\text { Running, } \\
\text { jogging, } \\
\text { orienteering }\end{array}$ & & & 1173 & $\begin{array}{l}\text { Patients with knee surgery 1991-93 } \\
\text { (primary reason TF OA), from national } \\
\text { register: } 52 \% \text { M, born 1921-38, } n=625\end{array}$ & $\begin{array}{l}\text { Randomly selected from population register, } \\
\text { born } 1921-38: 48 \% M, n=548\end{array}$ & $\begin{array}{l}\text { Physical activities aged 15- } \\
\text { 50yr, inc marathon, } \\
\text { jogging, orienteering } \\
\text { assessed by mailed } \\
\text { questionnaire }\end{array}$ \\
\hline $\begin{array}{l}\text { Muhlbauer } \\
2000^{9,37}\end{array}$ & $\begin{array}{l}\text { Retrospective } \\
\text { cohort }\end{array}$ & Triathlon & & $\geq 3$ & 36 & $\begin{array}{l}\text { 'Triathletes': recruitment unclear, } \\
50 \% \mathrm{M} \text {, mean age } 27.4(\mathrm{M}), 26.1(\mathrm{~F}) \text {, } \\
\mathrm{n}=18\end{array}$ & $\begin{array}{l}\text { 'Controls': recruitment unclear, 'physically } \\
\text { inactive', } 50 \% \mathrm{M} \text {, mean age } 22.2(\mathrm{M}), 22.3 \\
(\mathrm{~F}), \mathrm{n}=18\end{array}$ & $\begin{array}{l}\text { Trained for triathlon } \\
\geq 10 \mathrm{hr} / \mathrm{wk} \text { for } \geq 3 \mathrm{yr}\end{array}$ \\
\hline $\begin{array}{l}\text { Manninen } \\
2001^{33}\end{array}$ & Case-control & Running & NR & $\mathrm{n} / \mathrm{a}$ & 907 & $\begin{array}{l}\text { Patients with knee prosthetic surgery } \\
\text { (primary reason TF OA), from national }\end{array}$ & $\begin{array}{l}\text { Randomly selected from population register: } \\
48 \% \mathrm{M} \text {, age- and sex-matched, mean ages }\end{array}$ & $\begin{array}{l}\text { Lifetime recreational } \\
\text { exercise, inc running, }\end{array}$ \\
\hline
\end{tabular}




\begin{tabular}{|c|c|c|c|c|c|c|c|c|}
\hline & & & & & & $\begin{array}{l}\text { register: } 20 \% \mathrm{M} \text {, mean ages }(\mathrm{M}, \mathrm{F}) \\
67.5,69.2\end{array}$ & $(\mathrm{M}, \mathrm{F}) 67.2,67.1, \mathrm{n}=548$ & assessed by interview \\
\hline $\begin{array}{l}\text { Hohmann } \\
2005^{15}\end{array}$ & $\begin{array}{l}\text { Retrospective } \\
\text { cohort }\end{array}$ & $\begin{array}{l}\text { Long- } \\
\text { distance } \\
\text { running }\end{array}$ & $\begin{array}{l}\text { Competit } \\
\text { ive/elite }\end{array}$ & $\geq 5$ & 15 & $\begin{array}{l}\text { 'Advanced and professional' } \\
\text { marathon runners: recruitment } \\
\text { unclear: } 100 \% M \text {, median age } 34 \text { and } \\
33, n=8\end{array}$ & $\begin{array}{l}\text { 'Beginner' marathon runners: recruitment } \\
\text { unclear, } 86 \% M \text {, median age } 39, n=7\end{array}$ & $\begin{array}{l}\text { Reported running } \geq 5 \mathrm{yr} \\
\text { with marathon time }<4 \mathrm{hr}\end{array}$ \\
\hline $\begin{array}{l}\text { Thelin } \\
2006^{47}\end{array}$ & Case-control & Orienteering & Any & $\mathrm{n} / \mathrm{a}$ & 1473 & $\begin{array}{l}\text { Patients with diagnosis of TF OA } \\
\text { (Ahlback level } 3 \text { or more or knee } \\
\text { surgery or noted moderate cartilage } \\
\text { reduction or joint gap } \leq 3 \mathrm{~mm} \text { ) from } 6 \\
\text { hospital registers: } 43.2 \% \mathrm{M} \text {, mean age } \\
62.6, \mathrm{n}=778\end{array}$ & $\begin{array}{l}\text { Selected from population register: } 42.2 \% \mathrm{M} \text {, } \\
\text { mean age } 62.6 \text {, age-, sex- and municipality- } \\
\text { matched, } n=695\end{array}$ & $\begin{array}{l}\text { Reported regular } \\
\text { orienteering } \geq 1 \mathrm{yr} \text { since age } \\
16 y r \text {, assessed by mailed } \\
\text { questionnaire }\end{array}$ \\
\hline $\begin{array}{l}\text { Felson } \\
2007^{10}\end{array}$ & $\begin{array}{l}\text { Prospective } \\
\text { cohort }\end{array}$ & $\begin{array}{l}\text { Running or } \\
\text { jogging }\end{array}$ & NR & 8.75 & 1279 & $\begin{array}{l}\text { Participants in Framingham Offspring } \\
\text { cohort who reported jogging or } \\
\text { running: } 44 \% \mathrm{M} \text {, mean age } 53.2 \\
\mathrm{n}=1279 \text { (full sample) }\end{array}$ & $\begin{array}{l}\text { Participants in Framingham Offspring cohort } \\
\text { who reported never jogging or running: } 44 \% \\
M \text {, mean age } 53.2, n=1279 \text { (full sample) }\end{array}$ & $\begin{array}{l}\text { Reported ever being } \\
\text { exposed to } \\
\text { jogging/running }\end{array}$ \\
\hline $\begin{array}{l}\text { Mosher } \\
2010^{36}\end{array}$ & $\begin{array}{l}\text { Retrospective } \\
\text { cohort }\end{array}$ & $\begin{array}{l}\text { Long- } \\
\text { distance } \\
\text { running }\end{array}$ & NR & $\geq 5$ & 37 & $\begin{array}{l}\text { Marathon runners recruited from } \\
\text { clubs: } 59 \% M,(2 \text { age groups) mean } \\
\text { ages } 25.7 \text { and } 52.6, n=22\end{array}$ & $\begin{array}{l}\text { Community controls (excl if regularly ran over } \\
\text { past } 5 y r): 40 \% \mathrm{M} \text {, ( } 2 \text { age groups) mean ages } \\
28.4 \text { and } 54\end{array}$ & $\begin{array}{l}\text { Self-described marathon } \\
\text { runners, mean } \geq 10 \mathrm{mi} / \mathrm{wk} \\
\text { over prior } 5 \mathrm{yr}\end{array}$ \\
\hline
\end{tabular}

Abbreviations: $\mathrm{M}=$ male, $\mathrm{F}=$ female, $\mathrm{n} / \mathrm{a}=$ not applicable, $\mathrm{NR}=$ not reported, $\mathrm{CHD}=$ coronary heart disease, $\mathrm{OA}=$ osteoarthritis, $\mathrm{TF}=$ tibiofemoral, $\mathrm{TKR}=$ total $\mathrm{knee}$ replacement; ${ }^{*} \mathrm{Ambispective}=$ both retrospective and prospective data collection. ${ }^{* *}$ Sample size depends on comparison made. 
Findings from each study are summarised in Table 2.

\section{Diagnosis of knee OA}

188

Seven cohort studies included diagnosis as an outcome, three of which measured incidence prospectively. The diagnostic criteria used were different in almost every study (see Table 2). Of the

4 studies which reported formal statistical comparisons, three found no differences in knee OA diagnoses between groups, though two were small in size and likely under-powered ${ }^{21,39}$. The two large studies found: firstly, no difference in knee OA rates between runners and controls within the same cohort, over 8 years ${ }^{10}$; and secondly, significantly increased odds of knee OA diagnosis amongst elite orienteers compared to controls ${ }^{25}$.

One case control study ${ }^{47}$ identified cases of tibiofemoral OA diagnosis from 6 hospital registers (see Table 3). The findings indicated no significant difference in the odds of knee OA in patients who had previously participated regularly in orienteering.

One publication from a prospective cohort reported the results of a case-cohort analysis ${ }^{28}$. This result was not extracted because the analysis was not in keeping with the original study design.

\section{Radiographic/imaging markers}

Nine cohort studies examined radiographic outcomes: six measured osteophytes; one sclerosis; three assessed cartilage thickness, volume or joint surface area; one measured knee joint angle; one looked at joint space; and one study employed a composite score (Table 2). 
207 For all but two of these outcomes, no significant differences were reported. Lane $1986{ }^{26}$ found female, but not male, runners had a higher mean sclerosis score at baseline; and Muhlbauer $2000^{37}$ found male triathletes had a greater joint surface area than controls.

210 Two studies specifically used MRI to identify joint changes in response to jogging (30mins) ${ }^{36}$ or

211 running a marathon ${ }^{15}$. Hohmann 2005 was a small study with no comparison reported. Mosher

2122010 found a significant difference in femoral cartilage thickness between marathon runners and controls before, but not after, a 30-minute 'jog', and only amongst older participants.

214 No case control studies identified cases using radiographic markers of knee OA.

217 None of the cohort studies assessed this outcome.

218 Three case control studies identified cases of knee arthroplasty from hospital registers ${ }^{20,33,42}$. No formal comparison was made between runners or orienteers and controls in the Sandmark 1999 study, although crude numbers of participants reporting jogging were lower amongst cases than controls. The other two studies found no significant differences.

\section{Knee pain}

Three cohort studies assessed knee pain as an outcome. Two of the studies did not report comparisons ${ }^{18,39}$. The other study found no significant difference in the odds of knee pain between elite orienteers and controls ${ }^{25}$. 
230 Only two studies investigated knee-related function or disability as an outcome ${ }^{19,25}$. Only Kujala

2311999 presented formal statistical comparisons, showing no significant difference in the odds of knee

232 associated disability between male elite orienteers and controls.

233 No case control studies defined cases on the basis of knee-associated disability. 


\begin{tabular}{|c|c|c|c|c|c|c|c|}
\hline Study ID & Comparison & Outcome definition & Results & Risk of bias & & & $\begin{array}{l}\text { Knee injury } \\
\text { accounted for? }\end{array}$ \\
\hline & & & 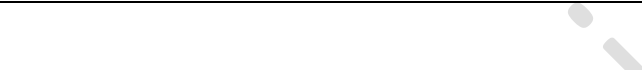 & Selectiont & $\begin{array}{l}\text { Comparability } \\
\ddagger\end{array}$ & Outcome§ & \\
\hline \multicolumn{8}{|c|}{ Diagnosis of knee $O A$} \\
\hline de Carvalho $^{7}$ & $\begin{array}{l}\text { Male club runners }(n=22) \text { vs } \\
\text { radiology patients }(n=25)\end{array}$ & $\begin{array}{l}\text { Joint space }<3 \mathrm{~mm} \text { or joint space } \leq 50 \% \\
\text { of other knee/opposite side or } \\
\text { sclerosing of articulation surface or } \\
\text { subchondral cysts }\end{array}$ & $\begin{array}{l}1 / 22 \text { runners, } 0 / 25 \text { controls had PF diagnosis. } 1 / 22 \\
\text { runners, } 1 / 25 \text { controls had TF diagnosis. No statistical } \\
\text { comparison. }\end{array}$ & $* * *$ & $*$ & $*$ & NR \\
\hline $\begin{array}{l}\text { Panush } \\
1986^{39}\end{array}$ & $\begin{array}{l}\text { Male runners ( } n=12) \text { vs } \\
\text { unspecified controls }(n=10)\end{array}$ & Ahlback grade $\geq 1$ & $\begin{array}{l}0 / 12 \text { runners, } 2 / 10 \text { controls. NS (statistical methods } \\
\text { NR). }\end{array}$ & $*$ & & $* *$ & $\mathrm{~N}$ \\
\hline Kujala $1994^{23}$ & $\begin{array}{l}\text { Male elite runners ( } n=163) \text { vs } \\
\text { community controls ( } n=1403)\end{array}$ & ICD code from hospital discharge report & $\begin{array}{l}2.5 \%(95 \% \mathrm{Cl} 0.7,6.3) \text { of runners and } 1.3 \%(95 \% \mathrm{Cl} 0.8, \\
2.0) \text { controls. No statistical comparison. }\end{array}$ & *** & * & ** & $\mathrm{N}$ \\
\hline Kujala $1994^{18}$ & $\begin{array}{l}\text { Male elite runners }(n=28) \text { vs } \\
\text { other elite sportsmen }(n=89)\end{array}$ & Kellgren-Lawrence grade $\geq 2$ & $\begin{array}{l}\text { 4/28 runners, } 1 / 29 \text { shooters, } 9 / 31 \text { soccer players, } 9 / 29 \\
\text { weightlifters. No statistical comparison. }\end{array}$ & $* * * *$ & $*$ & $* *$ & NR \\
\hline Felson $2007^{10}$ & $\begin{array}{l}\text { Runners/joggers ( } n=68 \text { ) vs non- } \\
\text { runners/joggers in Framingham } \\
\text { Offspring cohort }\end{array}$ & $\begin{array}{l}\text { Kellgren-Lawrence grade } \geq 2 \text { AND } \\
\text { reported knee pain, aching or stiffness } \\
\text { on most days }\end{array}$ & $\begin{array}{l}\text { Runners/joggers compared to controls: OR } 1.00(95 \% \\
\text { Cl 0.27, 3.68). NS (logistic regression) }\end{array}$ & **** & $* *$ & $* * *$ & $\mathrm{Y}$ - covariate \\
\hline $\begin{array}{l}\text { Konradsen } \\
1990^{21}\end{array}$ & $\begin{array}{l}\text { Male elite orienteers }(n=27) \text { vs } \\
\text { radiology patients }(n=27)\end{array}$ & Ahlback grade & $\begin{array}{l}\text { Grade } 3: 4 / 54 \text { runners' knees, 0/54 controls'. Grade 2: } \\
\text { 0/54 runners, } 0 / 54 \text { controls. Grade } 1: 31 / 54 \text { runners, } \\
\text { 27/54 controls. NS (Mann-Whitney) } \\
\text { NB - Excluded orienteers who were 'no longer active'. }\end{array}$ & ** & ** & ** & NR \\
\hline Kujala $1999^{25}$ & $\begin{array}{l}\text { Male elite orienteers }(n=264) \text { vs } \\
\text { community controls }(n=179)\end{array}$ & Self-report & $\begin{array}{l}\text { Runners compared to controls (OR 1.79; } 95 \% \mathrm{Cl} 1.10 \text {, } \\
\text { 3.54). (logistic regression) }\end{array}$ & *** & $* *$ & ** & $\mathrm{N}$ \\
\hline \multicolumn{8}{|c|}{ Radiographic markers } \\
\hline \multicolumn{8}{|l|}{ Osteophytes } \\
\hline $\begin{array}{l}\text { de Carvalho } \\
1977^{7}\end{array}$ & $\begin{array}{l}\text { Male club runners }(n=22) \text { vs } \\
\text { radiology patients }(n=25)\end{array}$ & Dichotomous (presence $\mathrm{Y} / \mathrm{N}$ ) & $\begin{array}{l}\text { 1/22 runners, } 3 / 25 \text { controls had unilateral PF } \\
\text { osteophytes. } 9 / 22 \text { runners, } 9 / 25 \text { controls had bilateral. } \\
5 / 22 \text { runners, } 4 / 25 \text { controls had unilateral TF } \\
\text { osteophytes. } 9 / 22 \text { runners, } 6 / 25 \text { controls had bilateral. } \\
\text { No statistical comparison. }\end{array}$ & *** & * & * & NR \\
\hline Lane $1986^{29}$ & $\begin{array}{l}\text { Club runners }(n=28) \text { vs } \\
\text { community controls }(n=27)\end{array}$ & $\begin{array}{l}\text { Score (sum of scores for each spur, 0-3) } \\
\text { Change in score from baseline (1993- } \\
\text { 1984) }\end{array}$ & $\begin{array}{l}\text { Runners' mean score } 1.24 \text { (SE } 0.32 \text { ) vs controls } 1.13 \text { (SE } \\
0.42 \text { ). NS ( } t \text { test) } \\
\text { Runners' mean score change } 0.80 \text { (SE0.23) vs controls } \\
0.67 \text { (SE 0.32). NS ( } t \text { test) }\end{array}$ & ** & * & ${ }^{* * *}$ & $\mathrm{~N}$ \\
\hline $\begin{array}{l}\text { Panush } \\
1986^{39}\end{array}$ & $\begin{array}{l}\text { Male runners ( } n=12) \text { vs } \\
\text { unspecified controls }(n=10)\end{array}$ & Number & $\begin{array}{l}\text { Runners' mean 0.4 (SD 1.4) vs controls' 1.3 (SD 4.1). NS } \\
\text { (statistical methods not reported) }\end{array}$ & * & & ** & $\mathrm{N}$ \\
\hline $\begin{array}{l}\text { Konradsen } \\
1990^{21}\end{array}$ & $\begin{array}{l}\text { Male elite orienteers }(n=27) \text { vs } \\
\text { radiology patients }(n=27)\end{array}$ & Number & $\begin{array}{l}\text { Runners' median } 1 \text { (range } 0 \text { to } 3 \text { ) vs controls median } 1 \\
\text { (range } 0 \text { to 5). NS (Mann-Whitney) } \\
\text { NB - Excluded orienteers who were 'no longer active'. }\end{array}$ & ** & ** & ** & NR \\
\hline Kujala $1994^{24}$ & Male elite runners $(n=28)$ vs & Dichotomous ( $\geq 1$ osteophyte graded $\geq 2$ & $4 / 28$ runners, $1 / 29$ shooters, $9 / 31$ soccer players, & $* * * *$ & ** & * & $\mathrm{N}$ \\
\hline
\end{tabular}




\begin{tabular}{|c|c|c|c|c|c|c|c|}
\hline \multirow[t]{2}{*}{ Study ID } & \multirow[t]{2}{*}{ Comparison } & \multirow[t]{2}{*}{ Outcome definition } & \multirow[t]{2}{*}{ Results } & \multicolumn{3}{|l|}{ Risk of bias } & \multirow[t]{2}{*}{$\begin{array}{l}\text { Knee injury } \\
\text { accounted for? }\end{array}$} \\
\hline & & & & Selectiont & Comparability & Outcome§ & \\
\hline & other elite sportsmen $(n=89)$ & on 0-3 scale) & $\begin{array}{l}\text { 10/29 weightlifters. NS (runners vs shooters, } \\
\text { generalized Fisher's exact test) }\end{array}$ & & & & \\
\hline $\begin{array}{l}\text { Spector } \\
1996^{44}\end{array}$ & $\begin{array}{l}\text { Female elite runners ( } n=67) \text { vs } \\
\text { controls }(n=977)\end{array}$ & $\begin{array}{l}\text { Dichotomous ( }(\geq 1 \text { osteophyte graded } \\
\geq 1 \text { on } 0-3 \text { scale) }\end{array}$ & $\begin{array}{l}\text { 13/67 runners, } 145 / 977 \text { controls had TF osteophytes. } \\
30 / 67 \text { runners, } 60 / 215 \text { controls had PF osteophytes. } \\
\text { No statistical comparison }\end{array}$ & & ** & $* * *$ & $\mathrm{~N}$ \\
\hline $\begin{array}{l}\text { Spector } \\
1996^{44}\end{array}$ & $\begin{array}{l}\text { Female elite runners }(n=67) \text { vs } \\
\text { elite tennis players }(n=14)\end{array}$ & $\begin{array}{l}\text { Dichotomous ( }(\geq 1 \text { osteophyte graded } \\
\geq 1 \text { on } 0-3 \text { scale) }\end{array}$ & $\begin{array}{l}\text { 13/67 runners, } 5 / 14 \text { tennis players had TF osteophytes. } \\
30 / 67 \text { runners, } 4 / 14 \text { tennis players had PF osteophytes. } \\
\text { No statistical comparison }\end{array}$ & ** & ** & *** & $\mathrm{N}$ \\
\hline \multicolumn{8}{|l|}{ Sclerosis } \\
\hline Lane $1986^{26}$ & $\begin{array}{l}\text { Club runners ( } n=41) \text { vs } \\
\text { community controls ( } n=41)\end{array}$ & $\begin{array}{l}\text { Score (sum of rating, } 0-3 \text {, for each 'area } \\
\text { of sclerosis'). At baseline only. }\end{array}$ & $\begin{array}{l}\text { Female runners mean score } 6.7 \text { (SE 0.5) vs controls } 5.1 \\
\text { (SE 0.3). } p<0.05 \text { ( } t \text { test). Male runners mean } 5.5 \text { (SE 0.4) } \\
\text { vs controls } 5.5 \text { (SE 0.5). NS ( } t \text { test) }\end{array}$ & * & * & $*$ & $\mathrm{~N}$ \\
\hline Cartilage & & & 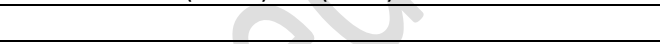 & & & & \\
\hline $\begin{array}{l}\text { Panush } 1986 \\
39\end{array}$ & $\begin{array}{l}\text { Male runners ( } n=12) \text { vs } \\
\text { unspecified controls }(n=10)\end{array}$ & $\begin{array}{l}\text { Sum of thickness }(\mathrm{mm}) \text { both knees, } \\
\text { radiograph }\end{array}$ & $\begin{array}{l}\text { Mean medial thickness } 5.18 \mathrm{~mm} \text { (SD 0.71) runners vs } \\
4.94 \mathrm{~mm} \text { (SD 1.12) controls. Lateral thickness } 6.58 \mathrm{~mm} \\
\text { (SD 1.06) runners vs } 5.85 \mathrm{~mm} \text { (SD 1.08) controls. NS } \\
\text { (statistical methods NR) }\end{array}$ & $*$ & & $* *$ & $\mathrm{~N}$ \\
\hline $\begin{array}{l}\text { Konradsen } \\
1990^{21}\end{array}$ & $\begin{array}{l}\text { Male elite orienteers }(n=27) \text { vs } \\
\text { radiology patients }(n=27)\end{array}$ & $\begin{array}{l}\text { Cartilage height }(\mathrm{mm}) \text { at medial \& } \\
\text { lateral compartments, radiograph }\end{array}$ & $\begin{array}{l}\text { Median medial thickness } 4 \mathrm{~mm} \text { runners, } 4 \mathrm{~mm} \text { controls. } \\
\text { Median lateral thickness } 5 \mathrm{~mm} \text { runners, } 5.5 \text { in controls. } \\
\text { NS (Mann-Whitney) } \\
\text { NB - Excluded orienteers who were 'no longer active'. }\end{array}$ & ** & ** & ** & NR \\
\hline $\begin{array}{l}\text { Muhlbauer } \\
2000^{9}\end{array}$ & $\begin{array}{l}\text { Triathletes }(n=18) \text { vs unspecified } \\
\text { controls }(n=18)\end{array}$ & $\begin{array}{l}\text { Cartilage volume (ml) taken from MRI, } \\
\text { right knee }\end{array}$ & $\begin{array}{l}\text { Mean volume males } 25.3 \mathrm{ml} \text { triathletes, } 23 \mathrm{ml} \text { controls; } \\
\text { females } 18.9 \mathrm{ml} \text { triathletes, } 17.9 \mathrm{ml} \text { controls. NS } \\
\text { Mean cartilage thickness males } 1.99 \mathrm{~mm} \text { triathletes, } \\
2.01 \mathrm{~mm} \text { controls; females } 1.93 \mathrm{~mm} \text { triathletes, } 1.86 \mathrm{~mm} \\
\text { controls. NS } \\
\text { Knee joint surface area males } 120 \mathrm{~cm}^{2} \text { triathletes, } \\
110 \mathrm{~cm}^{2} \text { controls. } p<0.01 \text { (Mann-Whitney). Females } \\
95.2 \mathrm{~cm}^{2} \text { triathletes, } 88.9 \mathrm{~cm}^{2} \text { controls. NS }\end{array}$ & & * & * & Y - excluded \\
\hline \multicolumn{8}{|c|}{ Knee joint angle } \\
\hline $\begin{array}{l}\text { Hohmann } \\
2005^{15}\end{array}$ & $\begin{array}{l}\text { Advanced }(n=8) \text { vs beginner }(n=7) \\
\text { marathon runners }\end{array}$ & Knee joint angle $>2^{\circ}$, radiograph & $\begin{array}{l}\text { Varus knees } 4 / 7 \text { beginners, } 4 / 6 \text { advanced, } 1 / 2 \\
\text { professionals. Valgus knees } 1 / 7 \text { beginners, } 1 / 6 \\
\text { advanced and } 0 / 2 \text { professionals. No statistical } \\
\text { comparison. }\end{array}$ & * & * & ** & Y- excluded \\
\hline \multicolumn{8}{|l|}{ Joint space } \\
\hline Lane $1986^{29}$ & $\begin{array}{l}\text { Club runners ( } n=28) \text { vs } \\
\text { community controls }(n=27)\end{array}$ & $\begin{array}{l}\text { Joint space narrowing score in } 1993 \text { (0- } \\
12 \text { scale) } \\
\text { Change in score (1993-1984) }\end{array}$ & $\begin{array}{l}\text { Mean score } 1.12 \text { (SE 0.22) runners, } 1.32 \text { (SE 0.24) } \\
\text { controls. NS ( } \mathrm{t} \text { test) } \\
\text { Mean score change } 0.20 \text { (SE 0.10) runners, } 0.32 \text { (SE } \\
0.12 \text { ) controls. NS ( } \mathrm{t} \text { test) }\end{array}$ & ** & $*$ & *** & $\mathrm{N}$ \\
\hline Lane $1986^{5}$ & Club runners $(n=45)$ vs & Joint space width $(\mathrm{mm})$ & $1 / 45$ runners, $5 / 53$ controls had joint space $0 \mathrm{~mm}$ (or & ** & ** & *** & $\mathrm{Y}$ - covariate \\
\hline
\end{tabular}




\begin{tabular}{|c|c|c|c|c|c|c|c|}
\hline \multirow[t]{3}{*}{ Study ID } & \multirow[t]{2}{*}{ Comparison } & \multirow[t]{2}{*}{ Outcome definition } & \multirow[t]{2}{*}{ Results } & \multicolumn{3}{|l|}{ Risk of bias } & \multirow[t]{2}{*}{$\begin{array}{l}\text { Knee injury } \\
\text { accounted for? }\end{array}$} \\
\hline & & & & Selectiont & $\begin{array}{l}\text { Comparability } \\
\ddagger\end{array}$ & Outcome§ & \\
\hline & community controls $(n=53)$ & & $\begin{array}{l}\text { TKR). } 4 / 45 \text { runners, } 6 / 53 \text { controls had width } \leq 1 \mathrm{~mm} \text {. } \\
5 / 45 \text { runners, } 7 / 53 \text { controls had width } \leq 2 \mathrm{~mm} \text {. } 11 / 45 \\
\text { runners, } 12 / 53 \text { controls had width } \leq 3 \mathrm{~mm} \text {. No statistical } \\
\text { comparison. } \\
\text { Running not associated with joint space width }(-0.15 \\
(95 \% \mathrm{Cl}-0.71,0.41) \text {. NS (linear regression) }\end{array}$ & & & & \\
\hline \multicolumn{8}{|l|}{ Total knee score } \\
\hline $\begin{array}{l}\text { Lane } 1986^{5} \\
29\end{array}$ & $\begin{array}{l}\text { 1. Club runners ( } n=28) \text { vs } \\
\text { community controls }(n=27) \\
\text { 2. Club runners ( } n=45) \text { vs } \\
\text { community controls }(n=53)\end{array}$ & $\begin{array}{l}\text { Knee score (sum of scores for } \\
\text { osteophytes, joint space, sclerosis, cysts } \\
\text { or erosions) (0-66 scale) }\end{array}$ & $\begin{array}{l}\text { 1. TKS in } 1993 \text { not associated with pace per mile }(0.27 \\
\text { (SE 0.15). } p=0.088 \text { (stepwise linear regression) }{ }^{29} \\
\text { 2. Running not associated with TKS ( } 0.72(95 \% \mathrm{Cl}-1.64 \text {, } \\
\text { 3.08). NS (linear regression) }\end{array}$ & $* *$ & * & $* * *$ & 2. $\mathrm{Y}$ - covariate \\
\hline \multicolumn{8}{|c|}{ Radiographic outcomes in response to running } \\
\hline Joint effusion & & & 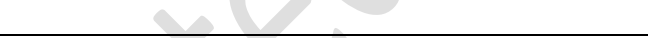 & & & & \\
\hline $\begin{array}{l}\text { Hohmann } \\
2005^{15}\end{array}$ & $\begin{array}{l}\text { Advanced }(n=8) \text { vs beginner }(n=7) \\
\text { marathon runners }\end{array}$ & $\begin{array}{l}\text { 'Stage } 2 \text { ' edema (T2 sequence by MRI): } \\
\text { pre- and post-marathon }\end{array}$ & $\begin{array}{l}\text { Pre-marathon, } 1 / 8 \text { advanced, } 0 / 7 \text { beginners. Post- } \\
\text { marathon, } 1 / 8 \text { advanced, } 5 / 7 \text { beginners. No statistical } \\
\text { comparison. }\end{array}$ & * & * & ** & $\mathrm{Y}$ - excluded \\
\hline Cartilage & & & 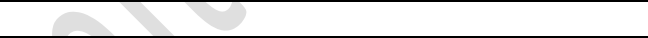 & & & & \\
\hline${ }_{36}^{\text {Mosher } 2010}$ & $\begin{array}{l}\text { Club marathon runners vs } \\
\text { community controls }\end{array}$ & $\begin{array}{l}\text { Femoral and tibial cartilage thickness } \\
\text { (mm) and cartilage T2, pre- and post- } \\
\text { 30-min jog, MRI }\end{array}$ & $\begin{array}{l}\text { Cartilage thicker in marathoners than controls. NS } \\
\text { except in older age group ( } \geq 46 \text { years) for femoral } \\
\text { cartilage pre-running (ANOVA, p value NR; group } \\
\text { means only presented graphically). } \\
\text { No difference runners and controls in T2 (values NR). }\end{array}$ & $* * *$ & * & ** & $\mathrm{Y}$ - excluded \\
\hline \multicolumn{8}{|l|}{ Knee pain } \\
\hline $\begin{array}{l}\text { Panush } 1986 \\
39\end{array}$ & $\begin{array}{l}\text { Male runners }(n=12) \text { vs } \\
\text { unspecified controls }(n=10)\end{array}$ & Unclear & $\begin{array}{l}\text { 0/12 runners, } 0 / 19 \text { controls reported pain. No } \\
\text { statistical comparison. } \\
\text { NB - sample likely biased due to drop-out ( } 29 \% \\
\text { runners reported pain at baseline). }\end{array}$ & $*$ & & $* *$ & $\mathrm{~N}$ \\
\hline $\begin{array}{l}\text { Kujala } 1994 \\
18\end{array}$ & $\begin{array}{l}\text { Male elite runners }(n=28) \text { vs } \\
\text { other elite athletes }(n=89)\end{array}$ & $\begin{array}{l}\text { Knee pain reported } \geq \text { monthly for prior } \\
12 \mathrm{mo}\end{array}$ & $\begin{array}{l}6 / 28 \text { runners, } 5 / 29 \text { shooters, } 14 / 31 \text { soccer players, } \\
8 / 29 \text { weightlifters reported pain. No statistical } \\
\text { comparison. }\end{array}$ & $* * * *$ & $*$ & $* *$ & $\mathrm{~N}$ \\
\hline $\begin{array}{l}\text { Kujala } 1999 \\
25\end{array}$ & $\begin{array}{l}\text { Male elite orienteers ( } n=264) \text { vs } \\
\text { community controls ( } n=179)\end{array}$ & $\begin{array}{l}\text { Knee pain reported by questionnaire, } \\
\geq \text { weekly for prior } 12 \mathrm{mo}\end{array}$ & $\begin{array}{l}\text { Compared to controls, runners OR } 1.75(95 \% \mathrm{Cl} 0.96 \text {, } \\
\text { 3.18). NS (logistic regression) }\end{array}$ & $* * *$ & $* *$ & $* *$ & $N$ \\
\hline \multicolumn{8}{|c|}{ Knee associated disability } \\
\hline $\begin{array}{l}\text { Kujala } 1994 \\
19\end{array}$ & $\begin{array}{l}\text { Male elite runners }(n=71) \text { vs } \\
\text { community controls }(n=460)\end{array}$ & $\begin{array}{l}\text { Score } \geq 3 \text { assessed on a 7-point scale, } \\
\text { based on } 7 \text { different activities. }\end{array}$ & $\begin{array}{l}5 / 71 \text { runners, } 59 / 460 \text { controls reported disability. No } \\
\text { statistical comparison. }\end{array}$ & *** & * & * & $\mathrm{N}$ \\
\hline $\begin{array}{l}\text { Kujala } 1999 \\
25\end{array}$ & $\begin{array}{l}\text { Male elite orienteers ( } n=264) \text { vs } \\
\text { community controls }(n=179)\end{array}$ & $\begin{array}{l}\text { Score } \geq 1 \text { on } 5 \text {-point scale, based on } 5 \\
\text { activities. }\end{array}$ & $\begin{array}{l}\text { Compared to controls, runners OR } 0.69(95 \% \mathrm{Cl} 0.39, \\
1.21) . \text { NS (logistic regression) }\end{array}$ & *** & $* *$ & $* *$ & $\mathrm{~N}$ \\
\hline & & Pain or discomfort in knee(s) when & Compared to controls, runners OR $0.78(95 \% \mathrm{Cl} 0.43$, & & & & \\
\hline
\end{tabular}




\begin{tabular}{|c|c|c|c|c|c|c|}
\hline Study ID & Comparison & Outcome definition & Results & Risk of bias & & $\begin{array}{l}\text { Knee injury } \\
\text { accounted for? }\end{array}$ \\
\hline & & & & Selectiont $\begin{array}{l}\text { Comparability } \\
\neq\end{array}$ & Outcome§ & \\
\hline & & using stairs & 1.41). NS (logistic regression) & 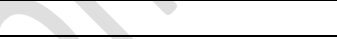 & & \\
\hline
\end{tabular}

Abbreviations: $\mathrm{Y}=$ yes, $\mathrm{N}=$ no, $\mathrm{n} / \mathrm{a}=$ not applicable, $\mathrm{NR}=$ not reported, $\mathrm{NS}=$ not significant, $\mathrm{Cl}=$ confidence intervals, $\mathrm{OR}=$ odds ratio, $\mathrm{SE}=$ standard error, $\mathrm{SD}=\mathrm{standard}$ deviation, $\mathrm{CHD}=\mathrm{coronary}$ heart disease, $\mathrm{OA}=$

240 Table 3 - Summary of case-control studies' findings

\begin{tabular}{|c|c|c|c|c|c|c|c|}
\hline \multirow[t]{2}{*}{ Study ID } & \multirow[t]{2}{*}{ Sport } & \multirow[t]{2}{*}{ Outcome definition } & \multirow[t]{2}{*}{ Results } & \multicolumn{3}{|c|}{ Risk of bias } & \multirow[t]{2}{*}{$\begin{array}{l}\text { Knee injury } \\
\text { accounted for? }\end{array}$} \\
\hline & & & & Selectiont & Comparability $\ddagger$ & Exposure§ & \\
\hline \multicolumn{8}{|c|}{ Diagnosis of knee $O A$} \\
\hline $\begin{array}{l}\text { Thelin } 2006 \\
47\end{array}$ & Orienteering & $\begin{array}{l}\text { TF OA diagnosis or knee } \\
\text { surgery or moderate cartilage } \\
\text { reduction or joint gap } \leq 3 \mathrm{~mm}- \\
\text { from hospital records }\end{array}$ & $\begin{array}{l}\text { Males who reported orienteering ( } \geq 1 \mathrm{yr} \text { since age } 16) \text { OR } 1.07(95 \% \mathrm{Cl} 0.62,1.82) \text {. } \\
\text { Females OR } 0.91(95 \% \mathrm{Cl} 0.34,2.45) \text {. NS (logistic regression) }\end{array}$ & *** & $* *$ & * & $\mathrm{N}$ \\
\hline \multicolumn{8}{|c|}{ Knee surgery for $O A$} \\
\hline $\begin{array}{l}\text { Kohatsu } \\
1990^{20}\end{array}$ & Running & $\begin{array}{l}\text { TKR for severe knee OA } \\
\text { ( } \geq \text { Grade } 3+\text { history knee pain) }\end{array}$ & 2/46 cases, 4/46 controls reported running. NS $\left(\mathrm{Chi}^{2}\right)$ & *** & * & * & $\mathrm{N}$ \\
\hline $\begin{array}{l}\text { Sandmark } \\
1999^{42}\end{array}$ & $\begin{array}{l}\text { Running or } \\
\text { orienteering }\end{array}$ & $\begin{array}{l}\text { Knee prosthetic surgery due to } \\
\text { primary TF OA }\end{array}$ & $\begin{array}{l}\text { Males: } 16 / 325 \text { cases, } 35 / 264 \text { controls reported jogging. } 30 / 325 \text { cases, } 27 / 264 \\
\text { controls orienteering. Females: } 8 / 300 \text { cases, } 14 / 284 \text { controls jogging. } 8 / 300 \\
\text { cases, } 5 / 284 \text { controls orienteering. No statistical comparison. }\end{array}$ & $* * * *$ & ** & ** & $\mathrm{Y}$ - excluded \\
\hline $\begin{array}{l}\text { Manninen } \\
2001^{33}\end{array}$ & Running & $\begin{array}{l}\text { Knee prosthetic surgery due to } \\
\text { primary TF OA }\end{array}$ & $\begin{array}{l}\text { Males who reported running OR } 0.26(95 \% \mathrm{Cl} 0.05,1.30) \text {, females } 0.70(95 \% \mathrm{Cl} \\
0.48,1.02) \text {. NS (logistic regression) }\end{array}$ & $* * *$ & $* *$ & *** & $\mathrm{Y}$ - covariate \\
\hline
\end{tabular}
and controls. ₹ Stars (of possible 2) awarded for control of confounding characteristics between groups. § Stars (of possible 3) awarded for ascertainment of exposure and non-response rate. 
Due to the heterogeneity of outcome definition and measurement of studies, only one meta-analysis was appropriate: this combined the case-control studies which identified cases of knee surgery due to OA (Figure 1). The combined odds ratio of undergoing knee surgery due to OA was $0.46(95 \% \mathrm{Cl}$

$2470.30,0.71)$ in runners or orienteers when compared with non-runners. The $I^{2}$ was $0 \%$, with $95 \% \mathrm{Cl}$ $0 \%$ to $73 \%$. No subgroup or sensitivity analyses were undertaken due to the small number of studies.

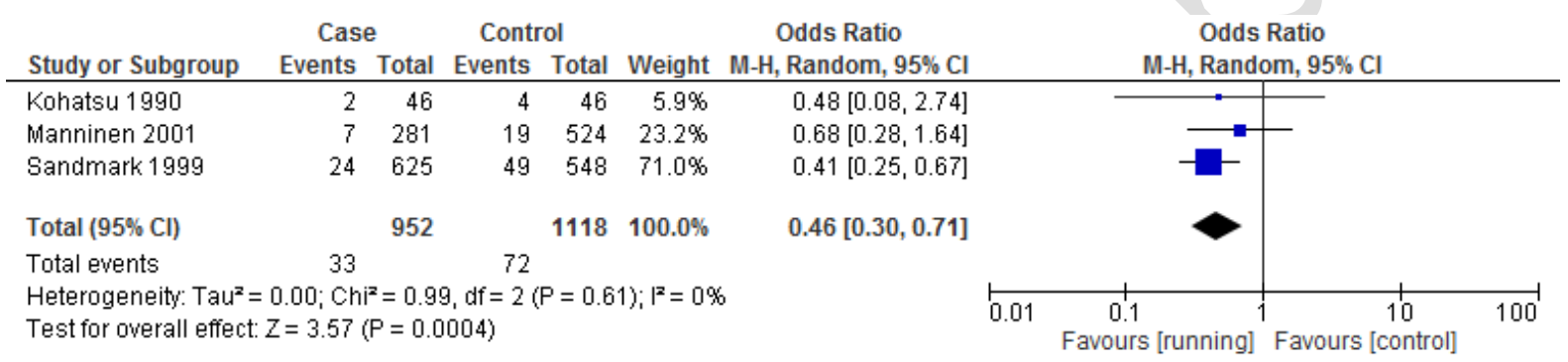

\section{Discussion}

This review has systematically gathered the peer-reviewed evidence regarding the role of running in

254 the development of knee OA. Five types of outcome relating to knee OA were considered: diagnosis, radiographic markers, surgery, and the symptomatic indicators of consistent knee pain and kneeassociated disability.

257 From this evidence, it is not possible to conclude if running was associated with diagnosis of knee OA, chiefly because the two best quality studies identified ${ }^{10,25}$ offered differing conclusions (one

259 finding no association and one finding a positive relationship). Nor was there evidence to support a

260 difference in radiographic or other imaging markers between runners and controls, with the exception being two studies which observed differences at baseline and only among subgroups 
262 (females, ${ }^{26}$ and older adults ${ }^{36}$ ). At follow-up, observed differences in these studies were not apparent. Evidence relating to symptomatic outcomes was sparse and therefore inconclusive.

However, a key finding of this review was the result of the meta-analysis, which was suggestive of around a $50 \%$ reduced odds of surgery due to OA amongst runners. The meta-analysis was based on case-control evidence and presents for the first time the odds ratio for the proportions reported in the Sandmark 1999 study. The meta-analysis result contradicts the apparent increased odds of OA diagnosis reported by Kujala 1999 as well as the conclusion of Felson 2007 in which no effect of running on OA diagnosis was found. There are a few possible explanations for these inconsistencies.

Firstly, the differences could be due to the different study designs, with the latter two studies employing prospective cohorts, whilst the meta-analysis used only retrospective data, which could reflect recall bias. No cohort evidence in this review investigated surgery as an outcome.

Secondly, the populations under investigation are not the same. Kujala 1999 was based on elite-level orienteers only, in contrast to the broader exposure levels implied by the samples of Felson 2007 and the case-control studies. Although this review was broad in its definition of running, it is possible that different types and performance levels of running relate differently to knee OA.

277 Thirdly, the outcomes are differently defined in these studies. Whilst surgery is often taken as a proxy for severe OA diagnosis, it could be speculated that the relationship between running and $\mathrm{OA}$ varies according to disease severity. So, for example, running could protect against OA progressing to severe stages, if not against diagnosis of mild or moderate OA. This remains conjecture at this point, due to the paucity of evidence.

The literature on overall leisure-time physical activity and knee joint replacement is a little more plentiful, but no more conclusive. Studies have variously reported no association ${ }^{1,2,33}$, a doseresponse increase in risk ${ }^{52}$, or a reduced risk but only at higher levels of activity (in men ${ }^{33}$ and in women ${ }^{1}$ ). At least two of these studies did not adjust for knee joint injury ${ }^{2,52}$. Manninen et al 
postulated that the relationship may be non-linear, as quadratic terms improved the fit of regression models ${ }^{33}$, implying a ' $U$ '-shaped curve. Comparing the findings of this review to the literature on physical activity, however, may not be useful, if, as previously discussed, running has a role independent of other sports and activities.

An important caveat in interpreting this evidence relates to its quality. Given the nature of observational studies, only low-to-moderate quality evidence could be expected ${ }^{13}$. However, the assessment of potential bias undertaken in this review indicated that many studies would be downgraded to low or very low quality. Just four studies were prospective (or ambispective) in design, and only one of these ${ }^{10}$ was a large, well-designed, prospective study addressing recreational (as well as more competitive) running, with controls recruited from same source, and appropriately adjusted analyses.

Most studies failed to take previous injury into account when looking at OA outcomes. Just two studies ${ }^{10,33}$ adjusted for knee injury in analyses, and four studies excluded participants with prior injury ${ }^{15,36,37,42}$. This is a key weakness in the evidence, given the strong association between injury and development of $\mathrm{OA}^{3,49}$. Without this adjustment, it cannot be judged whether the positive association reported by Kujala 1999, for example, was due to exposure to running (in the form of orienteering) or because elite-level orienteers were more prone to injury, therefore increasing their odds of OA diagnosis. This confounder could have influenced the results of many of the studies presented here.

The review by Shrier et al $^{43}$ concluded that running (at recreational or moderate level) does not cause or worsen OA. However, this included OA of any joint. The current review was unable to make a similar conclusion, due to the paucity of and contradictions in the evidence relating specifically to knee OA. Another more recent review ${ }^{8}$ reported increased odds for elite-level runners. However this was based only on two papers ${ }^{23,24}$, and the synthesis of data was methodologically flawed: firstly, the prevalence rates of the two papers were combined, despite both papers including runners 
from the same study, therefore effectively including the same participants twice; and, secondly, the authors calculated an additive odds ratio of the two studies, rather than reporting a pooled estimate from a meta-analysis).

In conducting this systematic review, the authors made every effort to minimise bias in identifying and collating the evidence: a pre-registered protocol was developed before searches began, and PRISMA guidelines have been followed. Independent reviewers assessed each article for eligibility and for risk of bias. In addition, the search was not limited by year or language, unlike many previous reviews. However, there are still limitations worth remarking on.

The meta-analysis included only a small number of studies, with odds ratios that represent unadjusted proportions (i.e. odds were not adjusted for confounding factors). Although the $\mathrm{I}^{2}$ indicated low heterogeneity, the upper $95 \% \mathrm{Cl}$ of the $\mathrm{I}^{2}$ is high (73\%), and the pooled estimate should be interpreted with caution. Furthermore, the pooled result chiefly reflects the findings of one study, Sandmark 1999, which has been heavily weighted by the Mantel-Haenszel method. However, the smaller studies included in the meta-analysis implied the same direction of effect, albeit with wide confidence intervals.

A strength of the review was including several types of outcome which related to knee OA. This allowed exploration into the possible differences in reported relationships according to outcome. That different measures may respond differently to an exposure is not a new idea. Urquhart et al ${ }^{50}$ offered a similar explanation for the contrasting findings of their review of physical activity and knee joint structures. The small number of studies relevant to each outcome in this review, however, makes it hard to establish if this is the case with running. cohorts had looked at arthroplasty as an outcome. In addition, most of the cohort studies recruited runners and controls from different sources, and were at risk of sampling bias, compounded by a 
failure to account for confounding factors. This review has also highlighted the dearth of evidence in recent years - just four publications in the past decade - which is surprising given the divergent (and often underpowered) findings previously. More well-designed, prospective evidence would help to clarify the contradictions observed.

\section{Conclusion}

341 This review was unable to conclude a role of running in the development of knee OA. Moderate to low quality evidence suggests both a positive association with OA diagnosis and a negative association with knee replacement surgery. Divergent results may be a reflection of methodological heterogeneity. Alternatively, they may be a result of a non-linear relationship between running exposure and risk of OA. It is surprising that research interest in this topic appears to have waned in recent years, particularly as participation rates continue to grow in many regions. This is in contrast to more studies investigating overall physical activity. However, activity- or sport-specific effects should not be ignored and the question of running remains clinically important. Given the many established beneficial effects of physical activity on other health outcomes, it is important to confidently inform the public about which forms of physical activity they can undertake without detriment to their musculoskeletal health. Currently, on the basis of published evidence, we are unable to offer advice about even one of the most popular activities, running. 
1. Ageberg E, Engstrom G, De Verdier MG, Rollof J, Roos EM, Lohmander LS. Effect of leisure time physical activity on severe knee or hip osteoarthritis leading to total joint replacement: a population-based prosective cohort study. BMC Musculoskeletal Disorders. 2012;13:73.

2. Apold H, Meyer HE, Nordsletten L, Furnes $O$, Baste V, Flugsrud G. Risk factors for knee

7. de Carvalho A, Langfeldt B. [Running practice and arthrosis deformans. A radiological assessment]. Ugeskr Laeger. 1977;139(44):2621-2622.

8. Driban J, Hootman JM, Sitler MR, Harris K, Cattano NM. Is participation in certain sports associated with knee osteoarthritis? A systematic review. Journal of Athletic Training 2015;50(2):000-000.

9. Eckstein F, Faber S, Muhlbauer R, et al. Functional adaptation of human joints to mechanical stimuli. Osteoarthritis \& Cartilage. 2002;10:44-50. 
10. Felson DT, Niu J, Clancy M, Sack B, Aliabadi P, Zhang Y. Effect of recreational physical activities on the development of knee osteoarthritis in older adults of different weights: the Framingham Study. Arthritis \& Rheumatism. 2007;57(1):6-12.

11. Goldring M, Goldring S. Osteoarthritis. J. Cell Physiol. 2007;213:626-634.

12. Gouttebarge V, Inklaar H, Backx F, Kerkhoffs G. Prevalence of osteoarthritis in former elite athletes: a systematic overview of the recent literature. Rheumatology International. 2015;35:405-418.

13. Guyatt $\mathrm{GH}$, Oxman $\mathrm{AD}$, Vist GE, et al. GRADE: an emerging consensus on rating quality of evidence and strength of reocmmendations. BMJ. 2008;336:924.

14. Higgins JPT, Green S. Cochrane Handbook for Systematic Reviews of Interventions: The Cochrane Collaboration; 2011.

15. Hohmann E, Wortler K, Imhoff AB. Osteoarthrose durch Langstreckenlaufen? Sportverl Sportschad. 2005;19:89-93.

16. Ioannidis J, Patsopoulos N, Evangelou E. Uncertainty in heterogeneity estimates in metaanalyses. BMJ. 2007;335:914-918.

17. Johnson V, Hunter D. The epidemiology of osteoarthritis. Best Practice \& Research in Clinical Rheumatology. 2014;28(1):5-15.

18. Kettunen JA, Kujala UM, Raty H, Sarna S. Jumping height in former elite athletes. European Journal of Applied Physiology \& Occupational Physiology. 1999;79(2):197-201.

19. Kettunen JA, Kujala UM, Kaprio J, Koskenvuo M, Sarna S. Lower-limb function among former elite male athletes. American Journal of Sports Medicine. 2001;29(1):2-8.

20. Kohatsu N, Schurman D. Risk factors for the development of osteoarthosis of the knee. Clin.Orthop. 1990;261:242-246.

21. Konradsen L, Hansen EM, Sondergaard L. Long distance running and osteoarthrosis. American Journal of Sports Medicine. 1990;18(4):379-381. 
22. Kujala U, Taimela S, Ilkka A-P, Orava S, Tuominen R, Myllynen P. Acute injuries in soccer, ice hockey, volleyball, basketball, judo, and karate: analysis of national registry data. BMJ. 1995;311:1465.

23. Kujala UM, Kaprio J, Sarna S. Osteoarthritis of weight bearing joints of lower limbs in former elite male athletes.[Erratum appears in BMJ 1994 Mar 26;308(6932):819]. BMJ. $1994 ; 308(6923): 231-234$.

24. Kujala UM, Kettunen J, Paananen $\mathrm{H}$, et al. Knee osteoarthritis in former runners, soccer players, weight lifters, and shooters. Arthritis \& Rheumatism. 1995;38(4):539-546.

25. Kujala UM, Sarna S, Kaprio J, Koskenvuo M, Karjalainen J. Heart attacks and lower-limb function in master endurance athletes. Medicine \& Science in Sports \& Exercise. 1999;31(7):1041-1046.

26. Lane NE, Bloch DA, Jones HH, Marshall WH, Jr., Wood PD, Fries JF. Long-distance running, bone density, and osteoarthritis. JAMA. 1986;255(9):1147-1151.

27. Lane NE, Bloch DA, Hubert HB, Jones H, Simpson U, Fries JF. Running, osteoarthritis, and bone density: initial 2-year longitudinal study. American Journal of Medicine. $1990 ; 88(5): 452-459$.

28. Lane NE, Michel B, Bjorkengren A, et al. The risk of osteoarthritis with running and aging: a 5-year longitudinal study. Journal of Rheumatology. 1993;20(3):461-468.

29. Lane NE, Oehlert JW, Bloch DA, Fries JF. The relationship of running to osteoarthritis of the knee and hip and bone mineral density of the lumbar spine: a 9 year longitudinal study. Journal of Rheumatology. 1998;25(2):334-341.

30. Lawrence RC, Felson DT, Helmick CG, et al. Estimates of the prevalence of arthritis and other rheumatic conditions in the United States, Part II. Arthritis \& Rheumatism. 2008;58(1):26-35.

31. Litwic A, Edwards M, Dennison E, Cooper C. Epidemiology and burden of osteoarthritis. $\mathrm{Br}$ Med Bull. 2013;105:185-199. 
32. Majewski M, Susanne H, Klaus S. Epidemiology of athletic knee injuries: a 10-year study. The Knee. 2006;13:184-188.

33. Manninen $P$, Riihimäki $H$, Heliövaara $M$, Suomalainen $O$. Physical exercise and risk of severe knee osteoarthritis requiring arthroplasty. Rheumatology. 2001;40(4):432-437.

34. Mantel N, Haenszel W. Statistical aspects of the analysis of data from retrospective studies of disease. Journal of the National Cancer Institute. 1959;22(4):719-748.

35. Moher D, Liberati A, Tetzlaff J, Altman DG, Group tP. Preferred reporting items for systematic reviews and meta-analyses: the PRISMA statement. BMJ. 2009;339:b2535.

36. Mosher TJ, Liu Y, Torok CM. Functional cartilage MRI T2 mapping: evaluating the effect of age and training on knee cartilage response to running. Osteoarthritis \& Cartilage. 2010;18(3):358-364

37. Muhlbauer R, Lukasz S, Faber S, Stammberger T, Eckstein F. Comparison of knee joint cartilage thickness in triathletes and physically inactive volunteers based on magnetic resonance imaging and three-dimensional analysis. American Journal of Sports Medicine. 2000;28(4):541-546.

38. Panush RS, Schmidt C, Caldwell JR, et al. Is running associated with degenerative joint disease? JAMA. 1986;255(9):1152-1154.

39. Panush RS, Hanson CS, Caldwell JR, Longley S, Stork J, Thoburn R. Is Running Associated with Osteoarthritis? An Eight-Year Follow-up Study. JCR: Journal of Clinical Rheumatology. 1995;1(1):35-39.

40. Raty HP, Kujala UM, Videman T, Impivaara O, Crites Battie M, Sarna S. Lifetime musculoskeletal symptoms and injuries among former elite male athletes. International Journal of Sports Medicine. 1997;18(8):625-632.

41. Roos EM. Joint injury causes knee osteoarthritis in young adults. Current Opinion in Rheumatology. 2005;17(2):195-200. 
42. Sandmark H, Vingard E. Sports and risk for severe osteoarthritis of the knee. Scand J Med Sci Sports. 1999;9:279-284.

43. Shrier I. Muscle dysfunction versus wear and tear as a cause of exercise related osteoarthritis: an epidemiological update. Br J Sports Med. 2004;38:526-535.

44. Spector TD, Harris PA, Hart DJ, et al. Risk of osteoarthritis associated with long-term weightbearing sports: a radiologic survey of the hips and knees in female ex-athletes and population controls. Arthritis \& Rheumatism. 1996;39(6):988-995.

45. Sterne JAC, Gavaghan D, Egger M. Publication and related bias in meta-analysis: power of statistical tests and prevalence in the literature. Journal of Clinical Epidemiology. 2000;53:1119-1129.

46. Review Manager (RevMan) [computer program]. Version 5.3. Copenhagen: The Nordic Cochrane Centre; 2014.

47. Thelin N, Holmberg S, Thelin A. Knee injuries account for the sports-related increased risk of knee osteoarthritis. Scandinavian Journal of Medicine \& Science in Sports. 2006;16(5):329333.

48. Thomas E, Peat G, Croft P. Defining and mapping the person with osteoarthritis for population studies and public health. Rheumatology. 2014;53:338-345.

49. Toivanen AT, Heliovaara M, Impivaara O, et al. Obesity, physically demanding work and traumatic knee injury are major risk factors for knee osteoarthritis - a population-based study with a follow-up of 22 years. Rheumatology. 2010;49:308-314.

50. Urquhart DM, Tobing JFL, Hanna FS, et al. What is the effect of physical activity on the knee joint? A systematic review. Medicine \& Science in Sports \& Exercise; 2011.

51. Vignon E, Valat JP, Rossignol M, et al. Osteoarthritis of the knee and hip and activity: a systematic international review and synthesis (OASIS). Joint, Bone, Spine: Revue du Rhumatisme. 2006;73(4):442-455. 
52. Wang $\mathrm{Y}$, Simpson JA, Wluka $\mathrm{AE}$, et al. Is physical activity a risk factor for primary knee or hip replacement due to osteoarthritis? A prospective cohort study. J Rheumatol. 2011;38(2):350-357.

53. Wells G, Shea B, O'Connell D, et al. The Newcastle-Ottowa Scale (NOS) for assessing the quality of nonrandomised studies in meta-analyses. Available at:

http://www.ohri.ca/programs/clinical epidemiology/oxford.asp. Accessed 26 Jan 2016.

486 